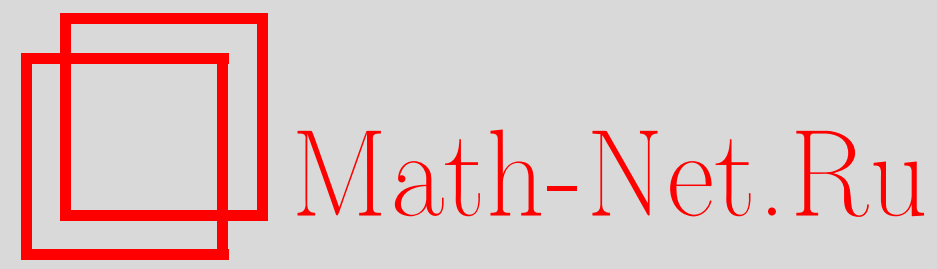

А. Б. Мухин, Аппроксимация локальных вероятностей сумм независимых случайных величин, Теория вероятн. $и$ ее примен., 1997, том 42, выпуск 4, 715-730

DOI: https://doi.org/10.4213/tvp2181

Использование Общероссийского математического портала Math-Net.Ru подразумевает, что вы прочитали и согласны с пользовательским соглашением http://www. mathnet.ru/rus/agreement

Параметры загрузки:

IP : 54.224 .135 .184

26 апреля 2023 г., 10:36:51

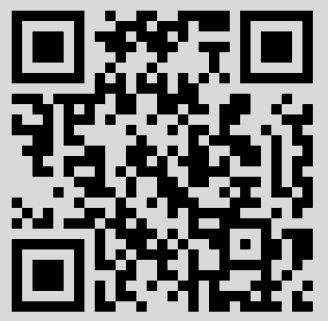


(C) $1997 \mathrm{r}$.

МУХин А. Б.

\section{АПІРОКСИМАЦИЯ ЛОКАЛЬНЫХ ВЕРОЯТНОСТЕЙ СУММ НЕЗАВИСИМЫХ СЛУЧАЙНЫХ ВЕЛИЧИН}

Рассматриваются суммы независимых случайных величин, нормированные распределения которых аппроксимированы соответствующим распределением или асимптотическим разложением. В этих условиях оценивается точность аппроксимации вероятностей попадания ненормированных сумм в ограниченную область. Оценки зависят от точности аппроксимации нормированных сумм и некоторых структурных характеристик слагаемых. В качестве следствий даются оценки скорости сходимости в локальных предельных теоремах о вероятности попадания сумм в ограниченную область для конкретных классов случайных величин.

Ключевые слова и фразы: суммы независимых случайных величин, локальные вероятности, локальные предельные теоремы, структурные характеристики распределений.

1. Введение. Пусть $S_{n}=\sum_{k=1}^{n} \xi_{k}$, где $\xi_{k}$ - вещественные, независимые для $k=1, \ldots, n$ случайные величины (с.в.); $\eta_{n}=B_{n}^{-1}\left(S_{n}-A_{n}\right)$, $A_{n} \in \mathbf{R}, B_{n} \rightarrow \infty$. Все пределы и асимптотические соотношения рассматриваются при $n \rightarrow \infty$. $G_{n}(\cdot)$ - аппроксимируюшие распределения, т.е. для некоторого класса множеств

$$
\delta_{n}(A) \equiv \mathbf{P}\left\{\eta_{n} \in A\right\}-G_{n}(A) \longrightarrow 0 .
$$

Будем всегда предполагать, что $G_{n}$ абсолютно непрерывны, причем для характеристической функщии (х.ф.)

$$
\left|\varphi_{n}(t)\right|=\left|\int_{\mathbf{R}} e^{i t x} G_{n}(d x)\right| \leqslant \varphi|t|^{-\beta}, \quad \varphi>0, \beta>1 .
$$

В качестве $G_{n}$ может выступать также конечная обобценная мера с $G_{n}(\mathbf{R})=1$ и соответствующим преобразованием Фурье-Стилтьеса.

Статья посвяшена оценкам

$$
\Delta_{n}(\lambda) \equiv \sup _{x \in \mathbf{R}}\left|\mathbf{P}\left\{S_{n} \in[x, x+\lambda]\right\}-G_{n}\left(\frac{[x, x+\lambda]-A_{n}}{B_{n}}\right)\right| .
$$

*Ташкентский государственный университет, математический факультет, Вузгородок, 700095 Ташкент, Узбекистан. 
В работах [1]-[4] изучены условия справедливости локальной предельной теоремы (л.І.т.) для областей, т.е. выполнения соотношений

$$
B_{n} \Delta_{n}(\lambda) \longrightarrow 0
$$

для постоянного $\lambda>0$ и $G_{n}=G_{1}$. Хорошо известны разнообразные результаты относительно оценок скорости сходимости в (1). Выделим работы [5], [6], в которых рассматриваются суммы одинаково распределенных конечномерных с.в., $G_{n}=\Phi-$ стандартное нормальное распределение. Даются оценки $\delta_{n}(A)$, зависящие от диаметра области $\rho_{A}$, что позволяет ощенить и $\Delta_{n}(\lambda)$. В частности, для одномерных с.в. с обшим распределением $F$ и конечным третьим моментом при $A_{n}=\mathbf{E} S_{n}$, $B_{n}^{2}=\mathbf{D} S_{n}$ для любого интервала $A$ получаем

$$
\left|\delta_{n}(A)\right| \leqslant c(F) n^{-1 / 2}\left(1+\rho_{A}\right)
$$

или

$$
\Delta_{n}(\lambda) \leqslant c(F) n^{-1 / 2}\left(1+\frac{\lambda}{\sqrt{n}}\right) .
$$

Здесь и далее $c(\cdot)$ - положительные постоянные, везде различные, зависяшие только от указанных в скобках параметров, $\boldsymbol{c}$ - абсолютные положительные постоянные. Очевидно, из (4) не следует (3), да и не может следовать, поскольку здесь не учитывается структура распределений слагаемых (решетчатость, нерешетчатость и т.д.). Некоторую информацию о скорости схоцимости в (3) можно извлечь из асимптотических разложений для нормированных распределений сумм (см., например, теорему 4 главы VI из [7]). Однако, при этом оценка $\Delta_{n}(\lambda)$ будет грубой даже при самых жестких условиях на структуру слагаемых и моменты.

Непосредственно оценкам скорости сходимости в (3) для частных случаев посвящены работы [8]-[10]. В [8] рассматриваются суммы независимых одинаково распределенных вещественных с.в., $G_{n}=G_{1}$ устойчивое распределение. В [9], [10] построены точные оценки в л.п.т. для областей в случае сумм конечномерных с.в., имеющих моменты порядка $2+\delta, \delta \in(0,1]$. В настояшей работе мы оцениваем $\Delta_{n}(\lambda)$ в общем случае, используя более эффективные структурные характеристики слагаемых по сравнению с [8]-[10].

2. Формулировка основных результатов. Основным условием далее будет следующее: существуют $\alpha>0, \tau>0, \nu \geqslant(\beta-1)^{-1}$ и последовательность $L_{n} \rightarrow 0$ такие, что

$$
\int_{|t| \leqslant \alpha L_{n}^{-\nu}}\left|f\left(\eta_{n}, t\right)-\varphi_{n}(t)\right| d t \leqslant \tau L_{n}
$$

где $f\left(\eta_{n}, t\right)-$ х.ф. $\eta_{n}$. Связь условия (5) с (1) очевидна. В частности, при выполнении (5) с $\nu \geqslant 1$ для любого конечного интервала $A$ име- 
ем $\delta_{n}(A)=O\left(L_{n}\right)$ (это следует из приводимой далее леммы 1 ). Если $\xi_{k}$ имеют конечные третьи моменты, $A_{n}=\mathbf{E} S_{n}, B_{n}^{2}=\mathbf{D} S_{n}, L_{n}=$ $B_{n}^{-3} \sum_{k=1}^{n} \mathrm{E}\left|\xi_{k}-\mathbf{E} \xi_{k}\right|^{3} \longrightarrow 0$, то (cм. [7], [11])

$$
\left|f\left(\eta_{n}, t\right)-e^{-t^{2} / 2}\right| \leqslant c L_{n}|t|^{3} e^{-t^{2} / 3}
$$

для $|t| \leqslant 1 /\left(4 L_{n}\right)$ и выполняется $(5)$. Далее в следствиях из общих теорем (п. 4) будут указаны другие классы последовательностей, для которых выполнено (5). В работе [12] близкое условие использовалось для построения оценок скорости сходимости в л.п.т. для плотностей.

Условие (5) и соотношение (1) связаны, как известно, с поведением распределений слагаемых «на хвостах». Для выполнения (3) и тем более оценок скорости сходимости в (3), очевидно, нужны дополнительные условия, исключаюшие сосредоточение распределений слагаемых и сумм на решетках. В связи с этим используем следуюшие характеристики распределений, изучавшиеся в [3], [4], [13]:

$$
H(\xi, d)=\mathbf{E}\left\langle\xi^{*} d\right\rangle^{2}
$$

где $\langle\cdot\rangle$ - расстояние от аргумента до ближайшего пелого числа, $\xi^{*}-$ симметризованная случайная величина. Очевидно, $H(\xi, d)=0$ тогда и только тогда, когда распределение $\xi$ решетчато с шагом $1 / d$ (считаем всегда $d>0)$. Положительность $H(\xi, d)$ при любом $d$ является критерием нерешетчатости. Укажем некоторые оценки отделенности $H(\xi, d)$ от нуля.

Если $\xi$ имеет ограниченную плотность $p(x) \leqslant A$ и конечный второй момент, то

$$
H(\xi, d) \geqslant \frac{c}{A^{2}\left(\mathrm{D} \xi+d^{-2}\right)} .
$$

Если $\xi$ абсолютно непрерывна, то

$$
H(\xi, d) \geqslant \frac{1}{12} \alpha\left(\mathfrak{N}, \frac{1}{2 d}\right)
$$

где $\alpha(\mathfrak{M}, N)$ - плотностные характеристики В. А. Статулявичюса [14].

Если выполняется условие Крамера: $\varlimsup$ lim $|f(\xi, t)|<1$ при $|t| \rightarrow \infty$, то $\inf _{d \geqslant \delta} H(\xi, d)>0$ для любого $\delta>0$ (верно и обратное утверждение).

Доказательства этих неравенств и некоторых других приведены в [13]. Следуюшее утверждение будет доказано в п. 4 настоящей статьи.

Eсли существуют рациональное число $\alpha \neq 0$ и иррачиональное алгебраическое число $\beta$ степени т такие, что $\mathrm{P}\left\{\xi^{*}=\alpha\right\}>0$ и $\mathrm{P}\left\{\xi^{*}=\right.$ $\beta\}>0$, то для пюбого $\delta>0$ при $m=2$

$$
\inf _{\delta \leqslant d \leqslant u} H(\xi, d) \geqslant c(F, \delta) u^{-2}
$$


а при $m>2$ для любого $\nu>2$

$$
\inf _{\delta \leqslant d \leqslant u} H(\xi, d) \geqslant c(F, \delta, \nu) u^{-\nu}
$$

Легко сформулировать исходное условие на языке разностей возможных значений не симметризованных случайных величин.

Положим

$$
H_{n}(d)=\sum_{k=1}^{n} H\left(\xi_{k}, d\right), \quad H_{n}=\inf _{1 \leqslant d \leqslant 2} H_{n}(d), \quad H_{n}^{*}=\inf _{d \geqslant 1} H_{n}(d)
$$

В [4] показано, что условие $H_{n} \rightarrow \infty$ является необходимым для справедливости усиленной л.п.т. для областей, а условие $H_{n}^{*} \rightarrow \infty$ необходимо для усиленной л.п.т. для плотностей. Там же имеются достаточные условия справедливости л.п.т., использующие $H_{n}(d)$.

Обозначим $\theta_{n}$ решение уравнения

$$
\theta=\exp \left\{-\inf _{1 \leqslant d \leqslant 1 / \theta} H_{n}(d)\right\}
$$

в области $0<\theta \leqslant 1$. Оно всегда сушествует, единственно и $\theta_{n} \leqslant$ $\exp \left(-H_{n}^{*}\right)$. Приведенные выше оценки $H(\xi, d)$ позволяют оценить $\theta_{n}$, в частности, если слагаемые имеют общее распределение $F$, то $\theta_{n} \rightarrow 0$ для нерешетчатого $F$ и $\theta_{n}=O\left(q^{n}\right), q=q(F)<1$, если $F$ удовлетворяет условию Крамера, т.е. $H_{1}^{*}>0$.

Еще обозначим $l_{n}=\alpha\left(L_{n}^{\nu} B_{n}\right)^{-1}, i_{n}=0$ при $l_{n}>2 \pi, i_{n}=1$ при $l_{n} \leqslant 2 \pi$. Для простоты формулировок считаем, что $B_{n} \geqslant 1, L_{n} \leqslant 1$ для любого $n \in \mathbf{N}$.

Теорема 1. Если в.ыполнены условия (2) и (5), то

$$
\Delta_{n}(\lambda) \leqslant a\left[\frac{\lambda L_{n}}{B_{n}}+\frac{\lambda i_{n}}{\sqrt{H_{n}}} \exp \left\{-\frac{l_{n}^{2} H_{n}}{4 \pi^{2}}\right\}+\theta_{n}\right]
$$

здесь и далее а и а от параметров в перечисленных условиях, в (10) можно взвть

$$
a=c\left(1+\tau+\frac{\varphi}{\beta-1}\left(1+\alpha^{1-\beta}\right)\right)
$$

Для справедливости неравенства (10), конечно, необязательно, чтобы $B_{n} \rightarrow \infty$ и $L_{n} \rightarrow 0$. Мы оставляем эти условия, чтобы сохранить преемственность с предшествуюшими результатами и поскольку неравенство (10) содержательно только при больших $B_{n}$ и малых $L_{n}$. Так как в (10) зависимость от распределений слагаемых полностью раскрыта, то указанная оценка $\Delta_{n}(\lambda)$ пригодна для случая, когда распределения $\xi_{k}$ зависят от $n$, т.е. для последовательности серий случайных величин. 
Следствия из теорем будут указаны далее. Сейчас приведем некоторые опенки $\Delta_{n}(\lambda)$ при цополнительных условиях.

Будем говорить, что быполнено условие $\mathrm{A}$, если для некоторых последовательностей $M_{n}$ и $B_{n}\left(0<M_{n}<B_{n}\right)$ существуют такие $\varepsilon>0$, $\rho \in(0,2]$, что для $u \geqslant M_{n}$

$$
B_{n}^{2}(u) \equiv \sum_{k=1}^{n} \int_{|x| \leqslant u} x^{2} F_{k}^{*}(d x) \geqslant \varepsilon u^{2-\rho} B_{n}^{\rho}
$$

где $F_{k}^{*}$ - симметризованные распределения $\xi_{k}$. Ограничиваясь значением. $\rho=2$, получаем более простое условие: $B_{n}^{2}\left(M_{n}\right) \geqslant \varepsilon B_{n}^{2}$. Последнее условие с постоянным $M_{n}=M$ и $B_{n}^{2}=\mathrm{D} S_{n}$ использовалось для построения достаточных условий справедливости л.п.т. для плотностей и ощенок скорости сходимости в них В. А. Статулявичусом [14]. Локальные предельные теоремы для областей с условием $\mathbf{A}$ рассматривались в [4] и пругих работах.

Теорема 2. Пусть выполнены условия (2), (5) и А с некоторой последовательностью $M_{n}$ и нормирующей последовательностью $B_{n}$ из (5). Tozda

$$
\Delta_{n}(\lambda) \leqslant \frac{a_{1}}{B_{n}}\left[\lambda L_{n}+M_{n} \theta_{n}^{*}\right]
$$

где $\theta_{n}^{*}-$ корень уравнения $\theta=\exp \left\{-\inf _{1 /\left(2 M_{n}\right) \leqslant d \leqslant 1 /\left(\theta M_{n}\right)} H_{n}(d)\right\}$, или

$$
\Delta_{n}(\lambda) \leqslant \frac{a_{2}}{B_{n}}\left[\lambda L_{n}+\left(\lambda \wedge M_{n}\right) M_{n} j_{n} \exp \left\{-\frac{H_{n}}{8 M_{n}^{2}}\right\}+\theta_{n}\left(M_{n}+1\right)\right]
$$

Здесь $a_{i}=a_{i}(\alpha, \beta, \varphi, \nu, \tau, \varepsilon, \rho), \lambda \wedge M_{n}=\min \left\{\lambda, M_{n}\right\}, j_{n}=0$ nрu $M_{n} \leqslant \frac{1}{2}$, $j_{n}=1$ nри $M_{n}>\frac{1}{2}, \theta_{n}-$ mo же, что и в теореме 1 .

Поскольку $\theta_{n}, \theta_{n}^{*} \leqslant 1$, то неравенства (13) и (14) полезны и без учета структуры слагаемых, в частности, из них следует (4).

С помощью леммы 5 из [16] неравенство (13) можно упростить:

Следствие. $В$ условиях теоремы 2

$$
\Delta_{n}(\lambda) \leqslant \frac{a_{1}}{B_{n}}\left[\lambda L_{n}+M_{n} \exp \left\{-H_{n}^{*} \wedge \frac{H_{n}}{16 M_{n}^{2}}\right\}\right] .
$$

Укажем еще одну оценку, особенно полезную при малых $\lambda$.

Теорема 3. Пусть выполнены условия (2), (5) и А. Положим $\sigma_{k}^{2}=$ $\mathbf{D} \xi_{k}$, если второй момент существует, $\sigma_{k}=\infty$ в противном случае. Тогда для любых $k_{1}<k_{2}<k_{3}<k_{4} \leqslant n, 0<\lambda \leqslant M_{n}$

$$
\Delta_{n}(\lambda) \leqslant \frac{a_{3}}{B_{n}}\left[\lambda L_{n}+\theta_{n}^{* *}\left(\lambda+\left\{\prod_{j=1}^{4} Q\left(\xi_{k_{j}}, \lambda\right)\left(\sigma_{k_{j}}+M_{n}\right)\right\}^{1 / 4}\right)\right]
$$


где $Q(\xi, \lambda)=\sup _{x} \mathbf{P}\{x \leqslant \xi \leqslant x+\lambda\}, \theta_{n}^{* *}-$ решение уравнения

$$
\theta=\exp \left\{-\inf _{1 /\left(2 M_{n}\right) \leqslant d \leqslant 1 /(\lambda \theta)} H_{n}(d)\right\} .
$$

3 а м е ч а н и е. Если фигурирующие в теореме 3 четыре с.в. $\xi_{k_{j}}$ имеют ограниченные плотности $p_{k_{j}}(x) \leqslant p$ и $\sigma_{k_{j}} \leqslant \sigma$, то

$$
\Delta_{n}(\lambda) \leqslant a_{4} \lambda B_{n}^{-1}\left[L_{n}+p\left(\sigma+M_{n}\right) \theta_{n}^{* *}\right]
$$

В этих условиях справедлива и соответствующая оценка в л.п.т. для плотностей.

\section{3. Доказательства теорем.}

Лемма 1. Пусть для последовательности случайньих величин $S_{n}$ (необязательно сумм независимых случайных величин) и некоторых $A_{n}$, $B_{n}$ и $G_{n}(\cdot)$, описанных во введении, выполняется (5). Тогда при $\lambda \geqslant 0$ и $0<\delta_{n} \leqslant 1 / l_{n}$ справедливы неравенства.

$$
\Delta_{n}(\lambda) \leqslant c \int_{l_{n} \leqslant t \leqslant 1 / \delta_{n}}\left|f_{n}(t)\right|\left[\left(\lambda+\delta_{n}\right) \wedge \frac{1}{t}\right] d t+a \frac{\lambda L_{n}+\delta_{n}}{B_{n}}
$$

где $f_{n}(t)=f\left(S_{n}, t\right)$, a определено соотношением (11).

В частности, при $\delta_{n}=1 / l_{n}$ из (15) следует оценка:

$$
\Delta_{n}(\lambda) \leqslant a\left(\lambda L_{n} B_{n}^{-1}+L_{n}^{\nu}\right)
$$

Д о к а з а т е ль с т в о л е м м ы 1 . Используем метод «сглаживания», для л.п.т., применявшийся в [2], [3], [9], [10]. Пусть $Q_{\varepsilon}(\cdot)-$ распределение с плотностью $q_{\varepsilon}(x)=\varepsilon\left(8 \pi x^{2}\right)^{-1} \sin ^{2}(8 x / \varepsilon)$. Как легко подсчитать, $Q_{\varepsilon}([-\varepsilon, \varepsilon]) \geqslant 1-1 /(4 \pi)$ и соответствующая х.ф. $f_{\varepsilon}(t)=0$ для $|t| \geqslant 16 / \varepsilon$ (см. $[15$, c. 96-97]). Далее будем пользоваться обозначениями: $A^{\varepsilon}=\{x:|x-y|<\varepsilon, y \in A\}, A^{-\varepsilon}=\mathbf{R} \backslash(\mathbf{R} \backslash A)^{\epsilon}, \partial A$ - граница множества $A$. Для краткости будем считать $A_{n}=0$. Положим $P_{n}(A)=\mathbf{P}\left\{S_{n} \in A\right\}, \bar{G}_{n}(A)=G_{n}\left(A / B_{n}\right), \varepsilon=\varepsilon_{n}=16 \delta_{n}$. Из неравенств сглаживания (см., например, лемму 11.4 или неравенство (11.25) из [15]) получаем

$$
\Delta_{n}(\lambda) \leqslant \frac{2 \pi}{2 \pi-1}\left(\gamma_{1}+\gamma_{2}+\gamma_{3}\right)
$$

где

$$
\begin{aligned}
& \gamma_{1}=\sup _{x \in \mathbf{R}} \bar{G}_{n}\left((\partial A)^{2 \varepsilon_{n}}+x\right), \quad A=[0, \lambda], \\
& \gamma_{2}=\sup _{x}\left|P_{n} * Q_{\varepsilon_{n}}\left(A^{\varepsilon_{n}}+x\right)-\bar{G}_{n} * Q_{\varepsilon_{n}}\left(A^{\varepsilon_{n}}+x\right)\right|, \\
& \gamma_{3}=\sup _{x}\left|P_{n} * Q_{\varepsilon_{n}}\left(A^{-\varepsilon_{n}}+x\right)-\bar{G}_{n} * Q_{\varepsilon_{n}}\left(A^{-\varepsilon_{n}}+x\right)\right| .
\end{aligned}
$$


Обозначая $g_{n}(x)$ плотность $G_{n}$ и учитывая (2), имеем

$$
\sup _{x} g_{n}(x) \leqslant \frac{1}{2 \pi} \int_{\mathbf{R}}\left|\varphi_{n}(t)\right| d t \leqslant \frac{1}{\pi}+\frac{\varphi}{\pi(\beta-1)}
$$

И

$$
\gamma_{1} \leqslant c\left(1+\frac{\varphi}{\beta-1}\right) \frac{\delta_{n}}{B_{n}} .
$$

Для любого распределения (конечной обобщенной меры) $F(\cdot)$ с абсолютно интегрируемой х.ф. (преобразованием Фурье-Стилтьеса) $f(t)$ можно записать

$$
F([x, x+\lambda])=\frac{1}{2 \pi} \int_{x}^{x+\lambda} \int_{\mathbf{R}} e^{-i t z} f(t) d t d z=\frac{1}{2 \pi} \int_{\mathbf{R}} f(t) \int_{x}^{x+\lambda} e^{-i t z} d z d t
$$

причем $\left|\int_{x}^{x+\lambda} e^{-i t z} d z\right| \leqslant \lambda \wedge 2 /|t|$. Отсюда

$$
\gamma_{2} \leqslant \frac{1}{\pi} \int_{\mathbf{R}}\left|f_{n}(t)-\varphi_{n}\left(t B_{n}\right)\right|\left|f_{\varepsilon_{n}}(t)\right|\left[\left(\lambda+\varepsilon_{n}\right) \wedge \frac{1}{|t|}\right] d t
$$

Обозначая через $I$ интеграл в $(15)$ и учитывая $(2),(5)$ и свойства $f_{\varepsilon}(t)$, получаем

$$
\begin{aligned}
\gamma_{2} \leqslant & \frac{\lambda+\varepsilon_{n}}{\pi B_{n}} \int_{|y| \leqslant \alpha L_{n}^{-\nu}}\left|f\left(\eta_{n}, y\right)-\varphi_{n}(y)\right| d y+c I \\
& +\frac{\lambda+\varepsilon_{n}}{\pi B_{n}} \int_{|y| \geqslant \alpha L_{n}^{-\nu}}\left|\varphi_{n}(y)\right| d y \\
\leqslant & c\left[\frac{\left(\lambda+\delta_{n}\right) \tau L_{n}}{B_{n}}+I+\frac{\left(\lambda+\delta_{n}\right) \varphi L_{n}^{\nu(\beta-1)}}{\alpha^{\beta-1}(\beta-1) \cdot B_{n}}\right] .
\end{aligned}
$$

Аналогично оценивается $\gamma_{3}$. Из (16), (17) и последних оценок следуeт (15).

Лемма 2. Для характеристической функиии суммы независимых случайных величин при любом $t \in \mathbf{R}$ справедливы неравенства

$$
\left|f_{n}(t)\right| \leqslant \exp \left\{-4 H_{n}\left(\frac{t}{2 \pi}\right)\right\} \text {. }
$$

$\Pi p u|t| \leqslant 2 \pi$

$$
\left|f_{n}(t)\right| \leqslant \exp \left\{-\frac{t^{2}}{4 \pi^{2}} H_{n}\right\}
$$

Доказательство следует из леммы 3 работы [3] и леммы 5 из [16].

Доказ а т л ль с т о т е о р е м ы 1 . В лемме 1 положим $\delta_{n}=$ $\theta_{n} /(2 \pi)$. Считаем $l_{n} \leqslant 2 \pi$ (в противном случае доказательство упрошается). Отметим, что $\theta_{n} \leqslant 1$. Оценим интеграл из (15):

$$
I \leqslant\left(\lambda+\delta_{n}\right) \int_{l_{n} \leqslant t \leqslant 2 \pi}\left|f_{n}(t)\right| d t+\frac{1}{2 \pi} \int_{2 \pi \leqslant t \leqslant 1 / \delta_{n}}\left|f_{n}(t)\right| d t=I_{1}+I_{2} .
$$

3 Теория вероятностей и ее применения, № 4 
Можно считать $H_{n} \geqslant 1$, иначе $\theta_{n} \geqslant 1 / e$ и оценка (10) тривиальна. Из (19) имеем

$$
I_{1} \leqslant\left(\lambda+\delta_{n}\right) \int_{t \geqslant l_{n}} \exp \left(-\frac{t^{2} H_{n}}{4 \pi^{2}}\right) d t \leqslant \frac{c\left(\lambda+\delta_{n}\right)}{\sqrt{H_{n}}} \exp \left(-\frac{l_{n}^{2} H_{n}}{4 \pi^{2}}\right) .
$$

Аналогично, из (18) получаем $I_{2} \leqslant\left(\theta_{n}\right)^{-1} \exp \left\{-4 \inf _{1 \leqslant d \leqslant 1 / \theta_{n}} H_{n}(d)\right\}=$ $\theta_{n}^{3}$. Собирая найденные оценки, получим (10).

Лемма 3. Пусть существуют $\varepsilon>0, \rho \in(0,2], h>0$ такие, что для $u \in\left[M_{n}, h B_{n}\right]$ выполнено (12) $\left(M_{n}\right.$ и $B_{n}$ те же, что в условии $\left.\mathbf{A}\right)$. Тогда для любого $\beta>0$

$$
\sup _{D \in \mathbf{R}} \int_{|t-D| \leqslant 1 / M_{n}}\left|f_{n}(t)\right|^{\beta} d t \leqslant \frac{a}{B_{n}},
$$

где $a=c / h+(c /(\rho \sqrt{\varepsilon} \beta))^{1 / \rho}$.

Доказательство без вычисления постоянной $a$ имеется в [3].

Док а з а те льс т в о те о рем ы 2 . Используем лемму 1 с $\delta_{n}=M_{n} \theta_{n}^{*}$

$$
\begin{aligned}
I & \leqslant\left(\lambda+\delta_{n}\right) \int_{l_{n} \leqslant t \leqslant \pi / M_{n}}\left|f_{n}(t)\right| d t+M_{n} \int_{\pi / M_{n} \leqslant t \leqslant 1 / \delta_{n}}\left|f_{n}(t)\right| d t \\
& =I_{1}+I_{2} .
\end{aligned}
$$

Из условия А стандартными выкладками, связанными с переходом к симметризованным с.в., легко получить оценку:

$$
\begin{aligned}
I_{1} & \leqslant\left(\lambda+\delta_{n}\right) \int_{l_{n} \leqslant t \leqslant \pi / M_{n}} \exp \left\{-c t^{2} B_{n}^{2}\left(\frac{\pi}{t}\right)\right\} d t \\
& \leqslant\left(\lambda+\delta_{n}\right) \int_{t \geqslant l_{n}} \exp \left\{-c \varepsilon t^{\rho} B_{n}^{\rho}\right\} d t=\frac{\lambda+\delta_{n}}{B_{n}} \int_{u \geqslant \alpha L_{n}^{-\nu}} \exp \left\{-c \varepsilon u^{\rho}\right\} d u \\
& \leqslant c(\varepsilon, \rho, \alpha, \nu) \frac{\lambda+M_{n} \theta_{n}^{*}}{B_{n}} \exp \left\{-\frac{c \varepsilon \alpha^{\rho}}{L_{n}^{\nu \rho}}\right\} \leqslant \frac{a\left(\lambda+M_{n} \theta_{n}^{*}\right)}{B_{n}} L_{n} .
\end{aligned}
$$

При оценке $I_{2}$ применим неравенство (18) и затем, разбивая область интегрирования, лемму 3 :

$$
\begin{aligned}
I_{2} \leqslant & M_{n} \exp \left\{-2 \inf _{1 /\left(2 M_{n}\right) \leqslant d \leqslant 1 /\left(\theta_{n}^{*} M_{n}\right)} H_{n}(d)\right\} \\
& \times \int_{\pi / M_{n} \leqslant t \leqslant 2 \pi /\left(\theta_{n}^{*} M_{n}\right)}\left|f_{n}(t)\right|^{1 / 2} d t \\
\leqslant & M_{n}\left(\theta_{n}^{*}\right)^{2} \frac{c}{\theta_{n}^{*}} \sup _{D} \int_{|t-D| \leqslant 1 / M_{n}}\left|f_{n}(t)\right|^{1 / 2} d t \leqslant \frac{a \theta_{n}^{*} M_{n}}{B_{n}} .
\end{aligned}
$$

Из (15), (20) и последних оценок получаем (13). 
Для доказательства (14) положим $\delta_{n}=\theta_{n} /(2 \pi)$ и оценим интеграл в лемме 1 следующим образом:

$$
\begin{aligned}
I \leqslant & \left(\lambda+\delta_{n}\right) \int_{l_{n} \leqslant t \leqslant \pi / M_{n}}\left|f_{n}(t)\right| d t \\
& +\left\{M_{n} \wedge\left(\lambda+\delta_{n}\right)\right\} \int_{\pi / M_{n} \leqslant t \leqslant 2 \pi}\left|f_{n}(t)\right| d t+c \int_{2 \pi \leqslant t \leqslant 1 / \delta_{n}}\left|f_{n}(t)\right| d t .
\end{aligned}
$$

Оценки получившихся интегралов не привоцим, поскольку они используют те же приемы, что и ранее.

Д ок азат ел ь с т о т е о е м ы 3. Положим в неравенстве (15) $\delta_{n}=\lambda \theta_{n}^{* *}$, при этом можно считать $\lambda>0$. Очевидно,

$$
\begin{aligned}
I & \leqslant\left(\lambda+\delta_{n}\right) \int_{l_{n} \leqslant t \leqslant \pi / M_{n}}\left|f_{n}(t)\right| d t+\left(\lambda+\delta_{n}\right) \int_{\pi / M_{n} \leqslant t \leqslant 1 / \delta_{n}}\left|f_{n}(t)\right| d t \\
& =I_{1}+I_{2}
\end{aligned}
$$

если $\lambda \theta_{n}^{* *} \geqslant M_{n} / \pi$, то $I_{2}=0$. Величина $I_{1}$ оценивается так же, как в теореме 2:

$$
I_{1} \leqslant \frac{a\left(\lambda+\delta_{n}\right) L_{n}}{B_{n}} \leqslant \frac{2 a \lambda L_{n}}{B_{n}} .
$$

Отметим, что $\bar{H}_{n}(d) \equiv H_{n}(d)-\sum_{j=1}^{4} H\left(\xi_{k_{j}}, d\right) \geqslant H_{n}(d)-1$. ІІри выполнении условия $\mathbf{A}$, обозначая $F_{k_{j}}^{*}(\cdot)$ симметризованное распределение $\xi_{k_{j}}$, имеем

$$
\begin{aligned}
\bar{B}_{n}^{2}(u) & \equiv B_{n}^{2}(u)-\sum_{j=1}^{4} \int_{|x| \leqslant u} x^{2} F_{k_{j}}^{*}(d x) \\
& \geqslant \varepsilon u^{2-\rho} B_{n}^{\rho}-4 u^{2}=\varepsilon u^{2-\rho} B_{n}^{\rho}\left[1-\frac{4}{\varepsilon}\left(\frac{u}{B_{n}}\right)^{\rho}\right] \\
& \geqslant \frac{\varepsilon}{2} u^{2-\rho} B_{n}^{\rho} \quad \text { длI } \quad u \in\left[M_{n},\left(\frac{\varepsilon}{8}\right)^{1 / \rho} B_{n}\right]
\end{aligned}
$$

Положим $f(t)=\prod_{j=1}^{4} f\left(\xi_{k_{j}}, t\right), \bar{f}_{n}(t)=f_{n}(t) f^{-1}(t)$.

Покрывая $\Omega=\left[\pi / M_{n}, 1 / \delta_{n}\right]$ интервалами $\Omega_{r}=\left[r / M_{n},(r+1) / M_{n}\right]$ и учитывая приведенные выше неравенства, с помощью лемм 2 и 3 оцеHим $I_{2}$ :

$$
\begin{aligned}
I_{2} & \leqslant 2 \lambda \sup _{\pi / M_{n} \leqslant t \leqslant 1 / \delta_{n}}\left|\vec{f}_{n}(t)\right|^{1 / 2} \sum_{r} \sup _{t \in \Omega_{r}}|f(t)| \int_{\Omega_{r}}\left|\vec{f}_{n}(t)\right|^{1 / 2} d t \\
& \leqslant \frac{a \lambda\left(\theta_{n}^{* *}\right)^{2}}{B_{n}} \sum_{r}\left|f\left(t_{r}\right)\right|, \quad t_{r} \in \Omega_{r} .
\end{aligned}
$$


Очевидно, функщии $g_{j}(t)=\left|f\left(\xi_{k_{j}}, t\right)\right|^{2}$ удовлетворяют условию Липшица $\left|g_{j}(t+s)-g_{j}(t)\right| \leqslant c \sigma_{k_{j}}|s|$. С помошью известного неравенства Эссеена для функций концентрации (см., например, $[7$, c. 56]) находим

$$
V_{j} \equiv \int_{\Omega} g_{j}(t) d t \leqslant \frac{c}{\lambda \theta_{n}^{* *}} Q\left(\xi_{k_{j}}, \lambda \theta_{n}^{* *}\right) .
$$

Теперь с помощью леммы 3 из [14] сумму в (23) можно оценить соответствующими интегралами:

$$
\begin{aligned}
\sum_{r} \prod_{j=1}^{4}\left|f\left(\xi_{k_{j}}, t_{r}\right)\right| & \leqslant \prod_{j=1}^{4}\left(\sum_{r} g_{j}^{2}\left(t_{r}\right)\right)^{1 / 4} \leqslant \prod_{j=1}^{4}\left[V_{j}\left(\sigma_{k_{j}}+M_{n}\right)\right]^{1 / 4} \\
& \leqslant \frac{c}{\lambda \theta_{n}^{* *}}\left[\prod_{j=1}^{4} Q\left(\xi_{k_{j}}, \lambda \theta_{n}^{* *}\right)\left(\sigma_{k_{j}}+M_{n}\right)\right]^{1 / 4}
\end{aligned}
$$

Из леммы 1 , неравенств (21)-(23) и последней оценки следует утверждение теоремы 3.

4. Частные случаи. Применим общие результаты к оценкам $\Delta_{n}(\lambda)$ для конкретных последовательностей $\xi_{k}$.

4.1. Следствие 1. Пусть $\xi_{k}$ одинаково распределень при $k=$ $1, \ldots, n$ (распределение может зависеть от $n$ ) и имеют моменты порядка $2+\delta, 0<\delta \leqslant 1 ; \gamma=\mathbf{E}\left|\xi_{k}-\mathbf{E} \xi_{k}\right|^{2+\delta} ; \sigma^{2}=\mathbf{D} \xi_{k} ; r=\left(\gamma / \sigma^{2}\right)^{1 / \delta}$. Тогда

$$
\begin{aligned}
\Delta_{n}(\lambda) & \equiv \sup _{x}\left|\mathbf{P}\left\{S_{n} \in[x, x+\lambda]\right\}-\Phi\left(\frac{[x, x+\lambda]-A_{n}}{B_{n}}\right)\right| \\
& \leqslant \frac{c(\delta)}{B_{n}}\left[\lambda L_{n}+(\lambda \wedge r) r \exp \left(-\frac{H_{1} n}{8 \cdot 4^{2 / \delta} r^{2}}\right)+(r+1) \exp \left(-H_{1}^{*} n\right)\right] .
\end{aligned}
$$

Здесь $A_{n}=\mathbf{E} S_{n}, B_{n}=\sigma \sqrt{n}, L_{n}=\gamma /\left(n^{\delta / 2} \sigma^{2+\delta}\right)$.

3 а м е ч а н и е. Отсюда следует (4). Если $H_{1}^{*}=0$, то вместо последнего слагаемого можно взять $(r+1) \theta_{n}$, где $\theta_{n}$ - решение уравнения $\theta=\exp \left\{-n \inf _{1 \leqslant d \leqslant 1 / \theta} H_{1}(d)\right\}$. Тогда для одинаково распределенных нерешетчатых с.в. с конечным третьим моментом получим оценку

$$
\Delta_{n}(\lambda) \leqslant \frac{c(F)}{\sqrt{n}}\left(\frac{\lambda}{\sqrt{n}}+\alpha_{n}\right)
$$

где $\alpha_{n} \rightarrow 0$ при $n \rightarrow \infty$ равномерно по $\lambda \geqslant 0$.

Нетрудно обобщить следствие 1 на случай различно распределенных с.в. с конечным моментом порядка $2+\delta$. 
Д ок азате ль с т в о сл е д с т в и я 1. Для х.ф. нормального закона выполняется (2) с $\beta=2$ и $\varphi=2 / e$. Имеются неравенства, обобщающие (6) при существовании моментов порядка $2+\delta, \delta \in(0,1]$. Например, в $[10$, с. 84$]$ показано, что при $|t| \leqslant 6\left(12 L_{n}\right)^{-1 / \delta}$

$$
\left|f\left(\eta_{n}, t\right)-\exp \left(-\frac{t^{2}}{2}\right)\right| \leqslant c L_{n}|t|^{2+\delta} \exp \left(-\frac{t^{2}}{3}\right),
$$

где $L_{n}=B_{n}^{-(2+\delta)} \sum_{k=1}^{n} \mathbf{E}\left|\xi_{k}-\mathbf{E} \xi_{k}\right|^{2+\delta}$. Из этого неравенства следует, что (5) выполняется с $\nu=1 / \delta, \alpha=6 \cdot 12^{-1 / \delta}, \tau=c(\delta)$.

Используя легко доказываемое неравенство

$$
B_{n}^{2}(u) \geqslant 2 B_{n}^{2}-4 u^{-\delta} \sum_{k=1}^{n} \mathbf{E}\left|\xi_{k}-\mathbf{E} \xi_{k}\right|^{2+\delta},
$$

легко проверить, что в условиях следствия выполняется условие $\mathbf{A}$ с $\rho=2, \varepsilon=1, M_{n}=4^{1 / \delta} r$.

Осталось использовать (14).

4.2. В случае одинаково распределенных $\xi_{k}$ (при всех $n$ и $k$ ) с конечным моментом порядка $2+\delta$, удовлетворяюших условию Крамера, из следствия 1 получаем: для любого фиксированного $\lambda \geqslant 0$

$$
\Delta_{n}(\lambda)=O\left(n^{-(1+\delta) / 2}\right),
$$

точнее, для $\lambda \geqslant 0$

$$
\Delta_{n}(\lambda) \leqslant c(F, \delta) n^{-(1+\delta) / 2}\left(\lambda+\theta^{n}\right),
$$

где $\theta=\theta(F)<1$. Справедливо более сильное утверждение.

Следствие 2. П̈усть $\xi_{k}$ имеют общее распределение $F$, удовлетворяющее условию Крамера. Условие

$$
\int_{|x| \geqslant z} x^{2} F(d x)=O\left(z^{-\delta}\right)
$$

при $z \rightarrow \infty$ является необходимьм и достаточным для справедливости $\kappa a \kappa(25), \max u(26)$.

Аналогичные результаты относительно л.п.т. для плотностей и решетчатых с.в. получены И. А. Ибрагимовым, отражены в [11] и хорошо известны.

Док аз а те ль с тв о сле д с т в я 2. Необходимость доказывается так же, как в теореме 4.5.1 из [11]. Достарочность докажем с помошью неравенства (14). Проверим выполнение условия (5) с $\nu=1 / \delta$, $L_{n}=n^{-\delta / 2}$. Следуя [11] и считая для простоты, что $\mathbf{E} \xi_{1}=0$, положим

$$
f\left(\xi_{1}, t\right)=\exp \left\{-\frac{\sigma^{2} t^{2}}{2}(1+\gamma(t))\right\}
$$


где $\gamma(t) \rightarrow 0$ при $t \rightarrow 0$. Выберем $\alpha$ так, чтобы $|\gamma(z)| \leqslant \frac{1}{2}$ для $|z| \leqslant \alpha / \sigma$. Тогда $|\gamma(t /(\sigma \sqrt{n}))| \leqslant \frac{1}{2}$ для $|t| \leqslant \alpha L_{n}^{-\nu}=\alpha \sqrt{n}$. При доказательстве теоремы 3.4.1 в [11] показано, что при выполнении (27) $\int_{0}^{x} t^{2}|\gamma(t)| d t=$ $O\left(x^{3+\delta}\right)$ для $x \rightarrow 0$. Отсюда теми же приемами, что и при доказательстве достаточности в лемме 3.4 .1 из [11], легко получить

$$
\int_{|t| \leqslant \alpha L_{n}^{-\nu}}\left|f\left(\eta_{n}, t\right)-\exp \left(-\frac{t^{2}}{2}\right)\right| d t=O\left(n^{-\delta / 2}\right)
$$

т.е. выполняется (5).

В условиях следствия существует такое $M=M(F)$, что

$$
B_{n}^{2}(u)=n \int_{|x| \leqslant u} x^{2} F^{*}(d x) \geqslant B_{n}^{2}=\sigma^{2} n
$$

для $u \geqslant M$, т.е. выполняется условие А с постоянным $M$. В силу теоремы 2 справедливо (14), из которого следует (25) и (26).

4.3. Пусть $\xi_{k}$ одинаково распределены, имеют нулевое математическое ожидание и моменты порядка $s \geqslant 3, \gamma_{\nu}=\mathbf{E}\left|\xi_{k}\right|^{\nu}, G_{n}(A)=$ $\int_{A}\left[\varphi(x)+\sum_{\nu=1}^{s-2} q_{\nu}(x) n^{-\nu / 2}\right] d x-$ стандартное асимптотическое разложение распределения нормированной суммы независимых случайных величин - см., например, [7, гл. VI, §1].

Следствие 3. В сформулированных выше условиях справедливы оченки:

$$
\begin{aligned}
\Delta_{n}(\lambda) & =\sup _{x}\left|\mathbf{P}\left\{S_{n} \in[x, x+\lambda]\right\}-G_{n}\left(\frac{[x, x+\lambda]}{B_{n}}\right)\right| \\
& \leqslant \frac{a}{\sqrt{n}}\left[\lambda n^{-(s-2) / 2}+(\lambda \wedge 1) e^{-a_{1} n}+e^{-H_{1}^{*} n}\right]
\end{aligned}
$$

где $a=a\left(s, \sigma, \gamma_{3}, \gamma_{s}\right), a_{1}=2^{-7} H_{1} \sigma^{4} \gamma_{3}^{-2}$.

Д о к а 3 а т е льст в о. Очевидно, (2) выполняется для любых $\beta>1$ и $\varphi=\varphi(\beta)$. Положим $\beta=s-1, L_{n}=n^{-(s-2) / 2}, \nu=(s-2)^{-1}$. Тогда справедливость неравенства (5) с $\tau=c(s) \gamma_{s} \sigma^{-s}, \alpha=\left(\sigma^{s} / \gamma_{s}\right)^{1 /(s-2)}$ следует из обычных оценок х.ф. нормированных сумм при наличии $s$-го момента, например, из леммы 4 гл. VI из [7]. В условиях следствия 3 выполняется условие А с $\varepsilon=1, \rho=2, M_{n}=4 \gamma_{3} \sigma^{-2}$ (см. доказательство следствия 1). Остается использовать неравенство (14).

4.4. Следствие 4. Пусть $\xi_{k}$ имеют общее распределение $F$ из области нормального притяжения устойчивого закона $G$ с показателем $\alpha \in(0,2]$. Существует абсолютный псевдомомент $\int_{\mathbf{R}}|x|^{T} \mid(F-$ $G)(d x) \mid<\infty$ порядка $r=1+[\alpha]$. Если выполняется условие Крамера, 
mо для пюбого $\lambda \geqslant 0$

$$
\begin{aligned}
\Delta_{n}(\lambda) & \equiv \sup _{x}\left|\mathbf{P}\left\{S_{n} \in[x, x+\lambda]\right\}-G\left(\frac{[x, x+\lambda]-A_{n}}{n^{1 / \alpha}}\right)\right| \\
& =O\left(n^{-(1+r-\alpha) / \alpha}\right) .
\end{aligned}
$$

Доказательство легко получается из теоремы 1. Условие А с постоянным $M$ также выполняется и с помощью теоремы 2 можно получить более точную, но громоздкую ощенку:

4.5. Пусть $\xi_{k}-$ с.в. с равномерными распределениями в интервалах длины $\Delta_{k}$. Положим

$$
B_{n}^{2}=\frac{1}{12} \sum_{k=1}^{n} \Delta_{k}^{2}, \quad r_{n}=\frac{\max _{1 \leqslant k \leqslant n} \Delta_{k}}{\sqrt{12} B_{n}}
$$

Ю. В. Прохоровым [17] доказано, что условие $r_{n} \rightarrow 0$ является необходимым и достаточным для справедливости как интегральной, так и л.п.т. для плотностей. Из теоремы 3 получаем

Следствие 5. $B$ сформулированных выше предположениях справедливо неравенство $B_{n} \Delta_{n}(\lambda) \leqslant c \lambda r_{n}$.

Доказательство легко получить, используя (6) и (7). Мы его не приводим, поскольку в данных условиях справедлива более сильная оценка для плотностей сумм:

$$
\left|B_{n} p\left(S_{n}, x\right)-\varphi\left(\frac{x-\mathbf{E} S_{n}}{B_{n}}\right)\right| \leqslant c r_{n} .
$$

Неравенство (28) с точным значением $c=51.45$ доказано Т. Л. Шервашидзе [18, теорема 4] при несущественном дополнительном условии $r_{n} \leqslant \sqrt{\frac{5}{12}}$. Покажем, что это условие можно опустить. Действительно, если оно не вынолняется, то

$$
\sup _{x} p\left(S_{n}, x\right) \leqslant \min _{1 \leqslant k \leqslant n} \sup _{x} p\left(\xi_{k}, x\right)=\frac{1}{\max _{1 \leqslant k \leqslant n} \Delta_{k}}<\frac{1}{\sqrt{5} B_{n}}
$$

и неравенство (28) для таких $r_{n}$ справедливо.

4.6. В приведенных следствиях условия на структуру слагаемых обеспечивались преимущественно выполнением условия Крамера или ограничениями на плотности абсолютно непрерывной компоненты. В случае дискретных или сингулярных распределений дать простые оценки $\Delta_{n}(\lambda)$ для достаточно широких классов последовательностей затруднительно. Проиллюстрируем возможность применения общих теорем в этом случае на примерах.

II р и м е $\mathrm{p}$ 1. $\xi_{k}$ имеют общее распределение $F$ с конечным третьим моментом (тогда $G_{n}=\Phi$ ). Если существуют рациональное число 
$\alpha \neq 0$ и иррациональное алгебраическое число $\beta$ степени $m \geqslant 2$ такие, что $\mathbf{P}\left\{\xi_{1}^{*}=\alpha\right\} \mathbf{P}\left\{\xi_{1}^{*}=\beta\right\}>0$, то для любого $\varepsilon>0$

$$
\Delta_{n}(\lambda) \leqslant \frac{c(F) \lambda}{n}+\frac{c(F, \varepsilon)}{n^{1-\varepsilon}} .
$$

Отметим, что использование нерешетчатости распределения слагаемых позволило здесь усилить общую оценку (4).

Для проверки (29) потребуются неравенства (8) и (9). Докажем их. Для определенности можно считать $0<\beta<\alpha$. В наших условиях имеем

$$
\begin{aligned}
H(\xi, d) & \geqslant c(F)\left(\langle\alpha d\rangle^{2}+\langle\beta d\rangle^{2}\right) \geqslant c(F)(\langle\alpha d\rangle+\langle\beta d\rangle)^{2} \\
& =c(F)(|\alpha d-s|+|\beta d-k|)^{2}=c(F) d^{2}\left(\left|\alpha-\frac{s}{d}\right|+\left|\beta-\frac{k}{d}\right|\right)^{2},
\end{aligned}
$$

где $s, k \in \mathbf{N}$, причем $s=s(\alpha, d) \sim \alpha d, k \sim \beta d$ при $d \rightarrow \infty$. Покажем, что при достаточно больших $d\left(d \geqslant d_{0}=d_{0}(F)\right)$

$$
H(\xi, d) \geqslant c(F) d^{2}\left(\beta-\frac{k}{s} \alpha\right)^{2}
$$

Пусть $|\alpha-s / d| \leqslant|\beta-(k / s) \alpha|$, иначе неравенство (30) очевидно. Поскольку $k / s \rightarrow \beta / \alpha<1$ при $d \rightarrow \infty$, то для $d$ достаточно больших

$$
\begin{aligned}
H(\xi, d) & \geqslant c(F) d^{2}\left(\left|\alpha-\frac{s}{d}\right|+\left|\beta-\frac{k}{s} \alpha+\frac{k}{s}\left(\alpha-\frac{s}{d}\right)\right|\right)^{2} \\
& \geqslant c(F) d^{2}\left(\left|\alpha-\frac{s}{d}\right|+\left|\beta-\frac{k}{s} \alpha\right|-\frac{k}{s}\left|\alpha-\frac{s}{d}\right|\right)^{2}
\end{aligned}
$$

Отсюда следует (30).

Из (30) в силу теоремы Лиувилля о приближении алгебраических чисел, уточненной К. Ротом [19], получаем $H(\xi, d) \geqslant c(F, \tau) d^{2} s^{-2 \tau}$, где $\tau=2$ при $m=2, \tau>2$ при $m>2$. Учитывая, что $s \sim \alpha d$ при $d \stackrel{\circ}{\rightarrow} \infty$, заключаем, что сушествует такое $d_{0}=d_{0}(F)$, что для $d \geqslant d_{0}$ и любого $\tau>2$ при $m>2$ и $\tau=2$ при $m=2$

$$
H(\xi, d) \geqslant c(F, \tau) d^{2-2 \tau} .
$$

В силу непрерывности $H(\xi, d)$ и нерешетчатости $F$ для любого $\delta>0$

$$
\inf _{\delta \leqslant d \leqslant d_{0}} H(\xi, d)=c\left(F, \delta, d_{0}\right)>0 .
$$

Отсюда и из (31) следуют неравенства (8) и (9). 
Выведем оценку (29) из (13). Как и в следствии 1, условие А здесь выполнено с постоянным $M$. Очевидно, корень $\theta_{n}^{*}$ уравнения

$$
\theta=\exp \left\{-n \inf _{1 /(2 M) \leqslant d \leqslant 1 /(\theta M)} H\left(\xi_{1}, d\right)\right\}
$$

в силу (8) и (9) не превосходит корня уравнения

$$
\theta=\exp \left\{-c(F, \nu) n \theta^{\nu}\right\}, \quad \nu>2,
$$

а в силу элементарного неравенства $e^{-x} \leqslant(u / e)^{u} x^{-u}$ для $x>0, u>0$ последний корень не превосходит корня уравнения

$$
\theta=c(F, \nu, u) n^{-u} \theta^{-\nu u}
$$

т.е. $\theta_{n}^{*} \leqslant c(F, \nu, u) n^{-u /(1+\nu u)}$. Выбирая $u$ достаточно большим, а $\nu$ достаточно близким к двум, получаем для любого $\varepsilon>0 \theta_{n}^{*} \leqslant c(F, \varepsilon) n^{-(1 / 2-\varepsilon)}$. Значения $B_{n}$ и $L_{n}$ указаны в следствии 1 . Теперь неравенство (29) следует из (13).

П р и м е р 2. $\xi_{k}$ имеют общее сингулярное распределение на $[0,1]$, построенное с помошью канторовского совершенного множества [20, c. 21]. Тогда

$$
\Delta_{n}(\lambda) \leqslant \frac{c}{\sqrt{n}}\left[\frac{\lambda}{\sqrt{n}}+\lambda^{c_{1}} \exp \left(-c_{0} n\right)\right],
$$

где $c_{1}=\log _{3} 2, c_{0}=\left(2 \pi^{2}\right)^{-1}\left(1-\exp \left(-\frac{1}{9}\right)\right)$.

Нетрудно показать, что в данном примере $Q\left(\xi_{1}, \lambda\right) \leqslant 2 \lambda^{c_{1}}$,

$$
\begin{aligned}
& H\left(\xi_{1}, d\right) \geqslant \frac{1}{2 \pi^{2}}\left(1-\exp \left(-d^{2}\right)\right) \quad \text { для } d \leqslant 1 \\
& H\left(\xi_{1}, d\right) \geqslant \frac{1}{2 \pi^{2}}\left(1-\exp \left(-\frac{1}{9}\right)\right) \quad \text { для } d \geqslant 1 .
\end{aligned}
$$

Поэтому (32) вытекает из теоремы 3. Отметим, что из теоремы 2 в данном случае получаются более грубые оценки в смысле зависимости oт $\lambda$.

\section{СПИСОК ЛИТЕРАТУРЫ}

1. Shepp L. A. A local limit theorem. - Ann. Math. Statist., 1964, v. 35, № 1, p. 419423.

2. Stone C. J. A local limit theorem for nonlattice multi-dimensional distribution functions. - Ann. Math. Statist., 1965, v. 36, № 2, p. 546-551.

3. Мухин $A$. Б. Локальные предельные теоремы для распределений сумм независимых случайных векторов. - Теория вероятн. и ее примен., 1984, т. XXIX, в. 2, c. $360-366$.

4. Мухин $А$. Б. Связь между локальными и интегральной предельными теоремами. - Теория вероятн. и ее примен., 1995, т. 40, в. 1, с. 96-110. 
5. von Bahr B. Multi-dimensional integral limit theorems. - Ark. Mat., 1967, v. 7, № 6, p. 71-88.

6. Сенатов $B: B$. Об одной оценке скорости сходимости в центральной предельной теореме. - В сб.: Вероятностные распределения и математическая статистика. Ташкент: Фан, 1986, с. 421-435.

7. Петров В. В. Суммы независимых случайных величин. М.: Наука, 1972, 414 с.

8. Володия $H$. А. Оценки в слабой форме локальной предельной теоремы для случая устойчивого распределения. - В сб.: Предельные теоремы и математическая статистика. Ташкент: Фан, 1976, с. 32-36.

9. Лария Ю. В. Об оценке скорости сходимости в локальной предельной теореме для областей. - Изв. АН У

10. Лария Ю. В. Аппроксимация распределений сумм независимых случайных векторов на ограниченных множествах. Дис.... канд. физ.-матем. наук. Ташкент: Ин-т матем. АН УзССР, 1989.

11. Ибрагимов И. А., Лияник Ю. В. Независимые и стащионарно связанные величины. М.: Наука, 1965, 524 с.

12. Macht $W$., Wolf $W$. The local limit problem and Holder-continuity. - Probab. Theory Relat. Fields, 1989, v. 82, № 2, p. 295-305.

13. Мухия А. Б. Сглаживание распределений сумм независимых случайных величин. Деп. ВИНИТИ, № 2861-В88, 1988, 51 с.

14. Статулнвиус $B$. A. Предельные теоремы для плотностей и асимптотические разложения для распределений сумм независимых случайных величин. - Теория вероятн. и ее примен., 1965, т. X, в. 4, с. 645-659.

15. Бхаттачария P. Н., Рао Р. Ранга. Аппроксимация нормальным распределением и асимптотические разложения. М.: Наука, 1982, 286 с.

16. Мухин $A$. Б. Локальные предельные теоремы для решетчатых случайных величин. - Теория вероятн. и ее примен., 1991, т. 36, в. 4, с. 660-674.

17. Прохоров Ю. В. Об одной локальной теореме. - В сб.: Предельные теоремы теории вероятностей. Ташкент: Фан, 1963, с. 75-80.

18. ШІерваиидзе T. Л. О равномерной оценке скорости сходимости в многомерной локальной предельной теореме для плотностей. - Теория вероятн. и ее примен., 1971, т. XVI, в. 4 , с. $765-767$.

19. Roth K. F. Rational approximation to algebraic number. - Mathematics, 1955, v. 2 , p. 1-20.

20. Лукач Е. Характеристические функции. М.: Наука, 1979, 424 с.

Поступила в редакцию

8.VII.1996 